\title{
Complicações gestacionais relacionadas ao uso de drogas por gestantes
}

\author{
Pregnancy complications related to drug use by pregnant women
}

Complicaciones del embarazo relacionadas con el consumo de drogas por mujeres embarazadas

Arthur Guimarães Rodrigues Dutra ${ }^{1 *}$, Andreza Gonçalves de Oliveira ${ }^{2}$, Beatriz Aragão Pascoal Carneiro $^{3}$, Emilly Cremasco Medeiros ${ }^{4}$, Katharine Guerra Cunha Veiga ${ }^{5}$, Raíssa Sanjuan Guedes Lima $^{3}$, Thais Kassia Brandalise ${ }^{6}$, Tiago Bernardes Coelho ${ }^{7}$, Victoria Cristina Rodrigues Ferraz ${ }^{8}$, Tatiane Carolina Batista Nacif Roza ${ }^{3}$.

\section{RESUMO}

Objetivo: Revisar e analisar as possíveis consequências relacionadas ao uso de drogas durante o período gestacional, a partir de uma revisão narrativa sobre o tema. Revisão bibliográfica: O uso de substâncias químicas lícitas e ilícitas durante o período gravídico pode ocasionar diversas complicações tanto à saúde materna quanto fetal. Essas complicações ocorrem devido à alta capacidade das substâncias atravessarem a barreira placentária e hematoencefálica, ocasionando efeitos adversos à saúde de ambos. O impacto gerado pelo uso de drogas no período gestacional está relacionando ao tipo de substância utilizada, dose e tempo de uso, entretanto entre as consequências mais frequentes estão a vasoconstrição placentária, 0 descolamento prematuro da placenta, o aborto espontâneo, o trabalho de parto prematuro e até mesmo a morte fetal. Considerações finais: A partir da análise dos efeitos de diversas substâncias químicas no organismo materno e fetal, reforça-se a importância orientar os profissionais de saúde sobre o adequado acompanhamento a essas gestantes usuárias de drogas, de forma a promover uma assistência pré-natal qualificada e a redução de danos para mãe e para o bebê.

Palavras-chave: Gravidez, Anormalidades induzidas por medicamentos, Drogas ilícitas, Usuários de drogas.

\begin{abstract}
Objective: Review and analyze the possible consequences related to drug use during the gestational period, based on a narrative review on the topic. Bibliographic Review: The use of legal and illegal chemical substances during the pregnancy period can cause several complications to both maternal and fetal health. These complications occur due to the high capacity of substances to cross the placental and blood-brain barrier, causing adverse health effects for both. The impact generated by the use of drugs in the gestational period is related to the type of substance used, dose and time of use, however among the most frequent consequences are placental vasoconstriction, premature placental detachment, miscarriage, premature labor and even fetal death. Final considerations: Based on the analysis of the effects of various chemical substances on the maternal and fetal organism, the importance of guiding health professionals on the proper
\end{abstract}

\footnotetext{
${ }_{1}^{1}$ Pontifícia Universidade Católica de Minas Gerais (PUC MINAS), Betim - MG.

*E-mail: arthu.dgk@gmail.com

2 Universidade Nove de Julho (UNINOVE), Guarulhos - SP.

${ }^{3}$ Centro Universitário de João Pessoa (UNIPE), João Pessoa - PB.

${ }^{4}$ Centro Universitário de Caratinga (UNEC), Caratinga - MG.

${ }^{5}$ Universidade Estácio de Alagoinhas (IDOMED), Alagoinhas - BA.

${ }^{6}$ Universidade de Caxias do Sul (UCS), Caxias do Sul - RS.

${ }^{7}$ Pontifícia Universidade Católica de Minas Gerais (PUC MINAS), Divinópolis - MG.

${ }^{8}$ Instituto Metropolitano de Ensino Superior (UNIVAÇO), Ipatinga - MG.
}

SUBMETIDO EM: 8/2021

ACEITO EM: 8/2021

PUBLICADO EM: 8/2021 
monitoring of these pregnant drug users is reinforced, in order to promote qualified prenatal care and reduction of harm to mother and baby.

Key words: Pregnancy, Abnormalities, Drug-induced, Illicit drugs, Drug users.

\section{RESUMEN}

Objetivo: Revisar y analizar las posibles consecuencias relacionadas con el consumo de drogas durante el embrazo, a partir de una revisión narrativa sobre el tema. Revisión bibliográfica: El uso de sustancias químicas legales e ilegales durante el período de embarazo puede causar varias complicaciones tanto para la salud materna como para la fetal. Estas complicaciones ocurren debido a la alta capacidad de las sustancias para atravesar la barrera placentaria y hematoencefálica, provocando efectos adversos para la salud de ambos. El impacto que genera el uso de drogas durante el embarazo está relacionado con la sustancia consumida, dosis y tiempo de uso, sin embargo, entre las consecuencias más frecuentes se encuentran la vasoconstricción placentaria, desprendimiento de placenta, aborto espontáneo, parto prematuro e incluso muerte fetal. Consideraciones finales: A partir del análisis de los efectos de diversas sustancias químicas sobre el organismo materno y fetal, se refuerza la importancia de orientar a los profesionales de la salud en el adecuado seguimiento de estas embarazadas usuarias de drogas, con el fin de promover una atención prenatal calificada y la reducción del daño a la madre y bebé.

Palabras clave: Embarazo, Anomalías inducidas por medicamentos, Drogas ilícitas, Consumidores de drogas.

\section{INTRODUÇÃO}

Há o conhecimento dentro da ciência de que diversos fármacos e substâncias podem gerar efeitos colaterais ao usuário, podendo esses efeitos serem benéficos ou maléficos. Quando maléficos, são chamados de efeitos adversos, os quais surgem por consequências do uso e, muitas vezes, do abuso de tais substâncias. Várias drogas, de natureza lícita, incluindo determinados fármacos como os opióides, quando utilizados em excesso ou sem prescrição médica por gestantes, acarretam impactos na saúde da mulher, do feto e também do recém-nascido. No entanto, o uso de substâncias consideradas tóxicas (como álcool e nicotina) e de drogas ilícitas (como cannabis, crack e cocaína) estão associados aos maiores níveis de complicações gestacionais (BECKER KL, 2017; TAMASHIRO EM, et al., 2020).

No grupo das gestantes, essas substâncias apresentam um alto potencial de causarem efeitos adversos, graças às suas propriedades farmacológicas que permitem a passagem pela barreira placentária e hematoencefálica. Logo, além de causar malefícios à mãe, causam prejuízos também ao filho. Nos Estados Unidos da América (EUA) foi encontrada prevalência do uso de drogas de abuso por, pelo menos, 4,4\% das mulheres grávidas com idade entre 15 a 44 anos. Já na Europa, 7,9\% das gestantes foram expostas a substâncias que atuam no sistema nervoso (dentre estas $4,4 \%$ eram usuárias de cocaína). No Brasil, apesar de muito corriqueiro o uso de drogas entre as gestantes, ainda são escassos estudos sobre o tema (OLIVEIRA TA, et al., 2016).

Dentre as complicações decorrentes do uso dessas substâncias, destacam-se, na gestante, a vasoconstrição placentária, o descolamento prematuro da placenta, o aborto espontâneo, o trabalho de parto prematuro, além de efeitos psicossociais, como estresse e condições de saúde mental, problemas financeiros e legais, apoio social reduzido e violência por parte do parceiro. Já no feto/neonato, podem ocorrer efeitos neurocognitivos de longo prazo, menor peso corporal ao nascer, restrição de crescimento intrauterino, síndrome da morte súbita do lactente, a síndrome de abstinência neonatal, além de desordens do espectro alcoólico fetal (COOK JL, et al., 2017).

Fatores socioeconômicos e socioculturais (violência doméstica na infância e adolescência; períodos de seis meses ou mais de desemprego; períodos autorrelatados de percepção de solidão durante a gravidez; 
etc.), exercem grande influência no contexto diário da gestante e também no uso de drogas. Pesquisas comprovaram que quanto menor o grau econômico e de escolaridade, maior a utilização de substâncias ilícitas dentre o grupo das mulheres grávidas. Além disso, propõe-se que este grupo apresentaria uma maior facilidade de acesso às drogas (SANCHÉZ-SAUCO MF, et al., 2019).

Diante do exposto, o presente estudo teve por objetivo revisar as possíveis consequências que o uso de algumas substâncias químicas, lícitas e ilícitas, podem desencadear no organismo da gestante e, ainda, as possíveis consequências na saúde fetal. Além disso, o estudo possui também como propósito gerar uma melhor visão aos profissionais de saúde quanto ao uso de drogas por parte das gestantes, proporcionandoIhes informações que auxiliem na orientação à gestante.

\section{REVISÃO BIBLIOGRÁFICA}

Rang HP, et al. (2016) definem um fármaco como uma substância química capaz de produzir algum efeito biológico ao ser administrado a um organismo vivo, desde que apresente estrutura conhecida pelo ser humano e não seja essencial na dieta desse. Já a droga, é caracterizada como uma substância que comumente causa reações maléficas ao ser administrada, como dependência e alterações de consciência.

Diversas são as substâncias conhecidas e listadas como drogas, tais quais: maconha, álcool, cocaína, cigarro, metanfetamina, dentre outras e, na sociedade atual, é bastante recorrente o consumo destas e conhecido seu alto potencial de gerar dependência. Apesar de estudos mostrarem que homens têm maior tendência no uso de drogas comparados às mulheres, tem sido frequente a identificação de gestantes usuárias de drogas pelos profissionais de saúde, o que representa uma grande preocupação, uma vez que esse comportamento é capaz de provocar consequências potencialmente graves tanto para a mãe quanto para a criança (ROCHA PC, et al., 2016).

O impacto do uso de drogas no período gestacional dependerá do tipo de substância utilizada, dose e tempo de uso. Os opioides podem comprometer a cognição, a memória, e levar à depressão respiratória e seu uso crônico pode resultar em desregulação endócrina, disfunções neurológicas e cardiovasculares (ELS C, et al., 2017). Dados epidemiológicos mostram que a ingestão de opioides durante a gravidez, sejam eles receitados ou não, quadruplicou entre os anos de 1999 a 2004 e que cerca de $56 \%$ das mortes por overdose de opioides entre mulheres ocorrem naquelas em idade reprodutiva. Além disso, até $35 \%$ das mulheres grávidas com transtorno do uso de opioides usam simultaneamente outras substâncias como a maconha, cocaína, benzodiazepínicos e tabaco (TOBON AL, et al., 2019).

As drogas ilícitas como cocaína, maconha, metanfetamina e crack geram transtornos de humor, alterações no estado de consciência e nas funções motoras. Elas debilitam o sistema imunológico, levando a predisposição de infecções, mas, na maioria das vezes, a toxicidade se manifesta em nível cardíaco e neurológico (ROY ND e GOSWAMI R, 2015).

A maconha, por exemplo, é a droga ilícita mais usada por gestantes (METZ TD e STICKRATH EH, 2015). Quanto à utilização de metanfetaminas, o número de usuários é estimado em mais de 30 milhões em todo o mundo, na sua maioria jovens em idade reprodutiva, tornando-a atualmente a droga ilícita mais usada depois da maconha (DINGER J, et al., 2017). Já o uso de cocaína vem aumentando anualmente, sendo que 15 a $17 \%$ dos usuários de cocaína são mulheres em idade fértil, o que, consequentemente pode acarretar aumento da prevalência do uso dessa droga durante a gravidez (WOULDES TA, et al., 2019; CEMBRANELLI E, 2012).

Já substâncias como o álcool e cigarro são legalizadas e de fácil acesso. O álcool é uma droga depressora do sistema nervoso, e pode levar a vários danos como alterações hepáticas, cardiovasculares e mentais. Estima-se que 46 milhões de mulheres no mundo sofram com transtornos relacionados ao consumo de álcool, com maior importância no grupo de gestantes e puérperas, uma vez que os resultados atingem diretamente a vida do feto (GONÇALVES LA, et al., 2020).

O uso de álcool também aparece associado ao fumo, uma vez que 31,3\% das gestantes adultas fumantes fizeram uso concomitante de cigarro e álcool (MOTTA KC e LINHARES MM, 2015). Um estudo brasileiro 
mostrou que cerca de $80 \%$ das mulheres fumantes avaliadas continuaram com tal hábito durante a gestação e que 6,1\% fizeram uso de álcool durante a gravidez, entretanto, as prevalências de uso encontradas na literatura variam muito, dependendo do tipo de estudo metodologia de pesquisa utilizados (KASSADA DS, et al., 2014).

Diversas condições já foram descritas como fatores de risco para o abuso de substâncias durante a gravidez e sua identificação precoce permite que se elaborem intervenções a fim de reduzir o risco maternofetal (CHANG G, 2021). Dentre esses fatores, o baixo nível de escolaridade, histórico de aborto anterior, ser gestante adolescente ou apresentar história de gravidez na adolescência, abuso sexual na infância, falta de apoio social e não coabitar com o companheiro associaram-se ao uso de substâncias lícitas e ilícitas no período gestacional (MOTTA KC e LINHARES MM, 2015; ROCHA PC, et al., 2016)

Uma vez que o número de mulheres em idade reprodutiva que usam drogas e se tornam dependentes destas substâncias aumenta a cada ano, deve-se considerar que esse quadro pode ser muito mais grave, visto que a dependência pode ser subdiagnosticada na gestação uma vez que a maioria das mulheres negam ou relatam um consumo menor das substâncias, por medo da repreensão e desaprovação pelo profissional de saúde e sociedade (KASSADA DS, et al., 2014).

\section{Consequências do uso crônico de substâncias}

\section{Álcool}

O efeito teratogênico do álcool pode causar manifestações maléficas em todos os estágios gestacionais, porém depende de fatores como a quantidade ingerida, a genética materna e fetal e a nutrição materna. Assim, o uso do álcool pode acarretar danos físicos ou mentais de acordo com o do grau de dependência alcoólica que a gestante está inserida e o abuso está associado com complicações como aborto, danos cerebrais ao feto e Síndrome Alcoólica Fetal (SAF) (CHANG G, 2021).

Assim, após ser ingerido pela gestante, o álcool atravessa a barreira placentária, expondo o feto às mesmas concentrações do sangue materno. No entanto, o feto não possui as enzimas necessárias para a transformação do álcool e seu metabólito, o acetaldeído, que é intensamente citotóxico, mutagênico e teratogênico a nível sanguíneo e está relacionado tanto à dependência, quanto aos efeitos teratogênicos. Logo, a SAF é uma doença que consiste em um conjunto de anomalias físicas, comportamentais e cognitivas e entre os principais sinais clínicos estão a microcefalia, problemas de comportamento do tipo cognitivo e psicossocial, retardo mental, o transtorno na linguagem e retardo no crescimento pré-natal e pós-natal (PINZON TE, et al., 2015).

Nesse sentido, por não existir um padrão de consumo dessa substância na gestação considerado seguro, é recomendada a abstinência entre gestantes e mulheres com intenção de engravidar (GONÇALVES LA, et al., 2020).

\section{Tabaco}

O consumo de tabaco é responsável por doenças cardíacas, doença pulmonar obstrutiva crônica, acidente vascular cerebral e câncer de pulmão. Logo, a redução do uso dessa substância é de grande importância para a saúde pública, visto que o tabagismo durante a gestação é o principal fator de risco prevenível para vários desfechos negativos, tanto para o bebê quanto para a gestante (DIAS-DAMÉ JL, et al., 2018).

Estudo mostrou que este fator de risco responde por $5 \%$ a $8 \%$ dos partos prematuros, $13 \%$ a $19 \%$ dos nascimentos com baixo peso, e $5 \%$ a $7 \%$ da síndrome da morte súbita do lactente (LUCCHESE L, et al., 2016). Além disso já foi comprovado que o uso do tabaco durante a gestação relaciona-se com riscos aumentados de gravidez ectópica, descolamento prematuro de placenta, ruptura das membranas, placenta prévia, problemas no desenvolvimento neurológico do feto e aborto. Outrossim o uso de tabaco durante a gestação aumenta significativamente o risco de neuroblastoma para o feto, que se trata de um tumor do sistema nervoso simpático originado da crista neural e é o tumor mais comum em crianças recém-nascidas (GONÇALVES LA, et al., 2020; MÜLLER-SCHULTE E, et al., 2018). 


\section{Opioides}

O uso de opioides na gestação pode acarretar resultados neonatais adversos como prematuridade, síndrome de abstinência, complicações respiratórias, baixo peso ao nascer, além de morte infantil. Um estudo recente utilizando dados da Nationwide Inpatient Sample, com mais de 56 milhões de mulheres americanas, demonstrou que as gestantes em uso de opioides, independente da dose, tiveram um aumento significativo nas chances de morte materna no hospital, parada cardíaca, restrição de crescimento intrauterino, descolamento prematuro da placenta, trabalho de parto prematuro, oligoidrâmnio, necessidade de transfusão, natimorto prematuro, ruptura de membranas e parto cesáreo em comparação com aquelas que não faziam uso de opioides (TOBON AL, et al., 2019).

\section{Cannabis}

A Cannabis, também conhecida como maconha ou marijuana, é derivada do delta-9-tetrahidrocanabinol (THC), sendo a droga mais consumida em todo o mundo, e também durante o período gestacional. Geralmente os riscos maternos estão associados ao modo em que ela é utilizada ou a sua capacidade de dependência que, quando comparada às demais drogas ilícitas, tem uma porcentagem menor de risco de adicção (WANG GS, 2019).

No entanto, os resultados sobre o uso da Cannabis ainda são conflitantes. Pesquisas observaram que em gestantes o consumo dessa droga teve associação com doenças psiquiátricas como ansiedade e depressão. Outros efeitos adversos na gestante estariam associados à abstinência, náuseas e vômitos, podendo provocar a Síndrome de Hiperêmese Canabinoide (CHANG G, 2021).

Além disso, essa droga atravessa a placenta e pode ser associada com restrição de crescimento fetal, descolamento prematuro de placenta, ruptura prematura de membrana, morte fetal e nascimento prematuro, principalmente em gestantes que fazem uso concomitante de cocaína e crack (METZ TD e STICKRATH EH, 2015; TAMASHIRO E, et al., 2020).

\section{Cocaína}

A cocaína é o estimulante mais potente, de origem natural, do sistema nervoso central (SNC). A sua extração provém da folha da planta andina coca e sabe-se que seu uso regula os circuitos cerebrais de forma que alguns neurotransmissores sejam liberados em excesso. Seu consumo tem como efeitos uma potente vasoconstrição, midríase, taquicardia, hipertensão e aumento da temperatura corporal, semelhante ao papel do sistema nervoso simpático no organismo humano. No SNC pode causar dores de cabeça, agitação psicomotora ou até mesmo convulsões (GORELICK DA, 2021).

A exposição materna à cocaína é associada à toxicidade cardiovascular, risco de aborto espontâneo, descolamento prematuro de placenta e hipertensão, simulando um quadro de pré-eclâmpsia. Estudos mostram uma relação entre o uso da cocaína e aumento significativo de riscos neonatais como baixo peso ao nascer, nascimento prematuro, peso reduzido ao nascer, fetos de baixo peso para a idade gestacional, bem como diminuição do comprimento e perímetro cefálico (CHANG G, 2021).

\section{Crack}

O crack é um subproduto da cocaína, comercializado no formato de pedras, que são fumadas em cachimbos. As mulheres representam cerca de $21 \%$ dos usuários de crack nas áreas de uso de drogas e há relatos que esse número vem aumentando. Quando avaliadas no período gravídico, estudos mostram que a gravidez pode não funcionar como uma mudança significativa na cessação do uso de drogas por essas mulheres, refletindo a vulnerabilidade das usuárias de crack (MARANGONI SR e OLIVEIRA MF, 2013; LIMBERGER J, et al., 2016).

O uso de crack por gestantes está associado a risco aumentado de descolamento prematuro da placenta, presença de mecônio no feto, ruptura prematura do saco amniótico, baixo peso ao nascer, parto prematuro e anormalidades geniturinárias e abdominais. Além disso, o uso regular de crack durante esse período aumenta a probabilidade de a criança desenvolver hipertensão mais tarde na vida e ter dificuldades tanto de concentração quanto prejuízos no desenvolvimento psicomotor (NARCOWICZ S, et al., 2013). 


\section{Metanfetaminas}

A metanfetamina (MA) (3,4-metilenodioximetanfetamina ou MDMA) é uma droga sintética, com potente ação estimulante do SNC, sendo também neurotóxica (CEMBRANELLI E, et al., 2012). O abuso de metanfetaminas durante a gravidez representa um problema emergente de saúde, entretanto, os estudos que analisam os resultados da gravidez com o uso de MA têm sido conflitantes. No entanto, já foi evidenciado que entre as gestantes que relataram uso dessa substância há riscos aumentados de hipertensão e préeclâmpsia, ruptura prematura de membranas, placenta prévia, descolamento prematuro da placenta, restrição de crescimento intrauterino, cardiomiopatias, baixo peso ao nascer, parto prematuro e morte intrauterina, em comparação com mulheres não usuárias de substâncias (DINGER J, 2017; WRIGHT TE, et al., 2016; PFLÜGNER A, et al., 2018.)

Quando comparadas usuárias de MA com mulheres usuárias de cocaína, todos esses riscos foram menores, com exceção do risco de hipertensão e pré-eclâmpsia que aumentou em relação à cocaína. Um estudo epidemiológico realizado nos EUA comparando os resultados de nascimentos de bebês afetados por metanfetamina e por opioides com outros partos hospitalares, encontrou, entre os nascimentos afetados por metanfetamina, taxas mais altas de pré-eclâmpsia ( $9,3 \%$ vs $4,4 \%$ opioide) e descolamento da placenta $(4,3 \%$ vs $3,1 \%$ opioide) (WOULDES TA, et al., 2019).

Sabe-se que a interrupção do uso dessas substâncias a qualquer momento durante a gravidez melhora os resultados do parto, portanto, os recursos devem ser direcionados ao tratamento da dependência e à promoção do cuidado pré-natal (WRIGHT TE, et al., 2016). Diante deste cenário, medidas conscientizantes com apoio educacional e midiático são importantes no manejo do problema (FORRAY A, 2016).

\section{CONSIDERAÇÕES FINAIS}

Além dos diversos prejuízos à mãe e ao feto relacionados ao consumo de substâncias lícitas e ilícitas durante a gestação, estas mulheres tendem a menor aderência aos cuidados de pré-natal e menor vínculo com a equipe de saúde. Além disso, o uso dessas substâncias durante o período gravídico e puerpério configura um grave problema de saúde pública devido ao alto risco de intercorrências obstétricas e neonatais. Desse modo, é de suma importância orientar os profissionais de saúde que fazem o acompanhamento prénatal sobre a importância de acolher, orientar e apoiar gestantes usuárias de drogas, de forma a promover uma assistência pré-natal qualificada e a redução de danos para mãe e para o bebê.

\section{REFERÊNCIAS}

1. BECKER KL. O efeito da interação social entre os jovens nas decisões de consumo de álcool, cigarros e outras drogas ilícitas. Estudos Econômicos, 2017; 47(1): 65-92.

2. CEMBRANELLI E, et al. Consequências do uso de cocaína e metanfetamina durante a gravidez. Revista da Federação Brasileira das Sociedades de Ginecologia e Obstetrícia, 2012; 40(5): 241-245.

3. CHANG G. Substance use during pregnancy: Overview of selected drugs. 2021. Disponível em: https://www.uptodate.com/contents/substance-use-during-pregnancy-overview-of-selected-drugs. Acesso em: 14 de junho de 2021.

4. COOK J, et al. Epidemiology and Effects of Substance Use in Pregnancy. Journal of obstetrics and gynaecology, 2017; 39(10): 906-915.

5. DIAS-DAMÉ JL et al. Cessação do tabagismo na gestação: estudo de base populacional. Revista de Saúde Pública, 2019; 53(3): 1-11.

6. DINGER J, et al. Methamphetamine Consumption during Pregnancy - Effects on Child Health. Pharmacopsychiatry, 2017; 50(3): 107-113.

7. ELS C, et al. Adverse events associated with medium- and long-term use of opioids for chronic non-cancer pain: an overview of Cochrane Reviews. Cochrane Database Of Systematic Reviews. 2017; 10(10): CD012509.

8. FORRAY A. Substance use during pregnancy. F1000Research, 2016; 887: 1-9.

9. GONÇALVES LA, et al. Rastreio do consumo de bebidas alcoólicas em gestantes. Revista Mineira de Enfermagem, 2020; 24: e-1322

10. GORELICK DA. Cocaine use disorder in adults: Epidemiology, pharmacology, clinical manifestations, medical consequences, and diagnosis. 2021. Disponível em: https://www.uptodate.com/contents/cocaine-use-disorder-inadults-epidemiology-pharmacology-clinical-manifestations-medical-consequences-and-diagnosis. Acesso em 25 de agosto de 2021. 
11. KASSADA DS, et al. Percepções e práticas de gestantes atendidas na atenção primária frente ao uso de drogas. Escola Anna Nery - Revista de Enfermagem, 2014; 18(3): 428-434.

12. LIMBERGER J, et al. Women users of crack: Systematic review of Brazilian literature. Jornal Brasileiro de Psiquiatria, 2016; 65(1): 82-88.

13. LUCCHESE L, et al. Fatores associados ao uso nocivo do tabaco durante a gestação. Acta Paulista de Enfermagem, 2016; 29(3): 325-331.

14. MARANGONI SR; OLIVEIRA MF. Fatores desencadeantes do uso de drogas de abuso em mulheres. Texto \& Contexto Enfermagem, 2013; 22(3): 662-670.

15. METZ TD, STICKRATH EH. Marijuana use in pregnancy and lactation: a review of the evidence. American Journal of Obstetrics \& Gynecology, 2015; 213(6): 761-778.

16. MOTTA KC, LINHARES MM. Perfil das gestantes usuárias de álcool/drogas e os efeitos na saúde e desenvolvimento dos filhos. Revista interação em psicologia, 2015; 19(1): 133-144.

17. MÜLLER-SCHULTE E, et al. Tobacco, alcohol and illicit drugs during pregnancy and risk of neuroblastoma: systematic review. Archives of disease in childhood. Fetal and neonatal edition, 2018; 103(5): F467-F473.

18. NARCOWICZ S, et al. Prenatal exposure to substance of abuse: A worldwide problem. Environment International, 2013; 54: 141-163.

19. OLIVEIRA TA, et al. Perinatal Outcomes in Pregnant Women Users of Illegal Drugs. Revista Brasileira de Ginecologia e Obstetrícia, 2016; 38: 183-188.

20. PINZÓN TE, et al. Tratamiento Estomatológico en el paciente adolescente con síndrome alcohólico fetal. Acta Pediatrica Mexicana, 2015; 36: 32- 35.

21. PFLÜGNER A, et al. Methamphetamine Consumption During Pregnancy and its Effects on Neonates. Klinische Padiatrie, 2018; 230(1): 31-38.

22. RANG HP, et al. Farmacologia. 8. ed. Rio de Janeiro: Elsevier, 2016; 1939 p.

23. ROCHA PC, et al. Prevalência e fatores associados ao uso de drogas ilícitas em gestantes da coorte BRISA. Cadernos de Saúde Pública, 2016; 32(1): e00192714.

24. ROY DN, GOSWAMI R. Drugs of abuse and addiction: A slippery slope toward liver injury. Chemico-biological interactions, 2016; 255: 92-105.

25. SÁNCHEZ-SAUCO MF, et al. Sociocultural aspects of drug dependency during early pregnancy and considerations for screening: Case studies of social networks and structural violence. Midwifery, 2019; 78: 123-130.

26. TAMASHIRO EM, et al. "Because of the baby": reduction on drug use during pregnancy. Revista Brasileira de Saúde Materno Infantil, 2020; 20(1): 319-323.

27. TOBON AL, et al. Opioid Use in Pregnancy. Current Psychiatry Reports, 2019; 21: 118.

28. WANG GS. Cannabis (marijuana): Acute intoxication. 2021. Disponível em: https://www.uptodate.com/contents/cannabis-marijuana-acute-intoxication. Acesso em 25 de agosto de 2021.

29. WOULDES TA, Stimulants: how big is the problem and what are the effects of prenatal exposure? Seminars in fetal \& neonatal medicine, 2019; 24(2): 155-160.

30. WRIGHT TE, et al. Methamphetamines and pregnancy outcomes. Journal of addiction medicine, 2015; 9(2): $111-117$. 